\title{
Noradrenaline Triggers Multivesicular Release at Glutamatergic Synapses in the Hypothalamus
}

\author{
Grant R. J. Gordon and Jaideep S. Bains \\ Hotchkiss Brain Institute and the Department of Physiology and Biophysics, University of Calgary, Calgary, Alberta, Canada, T2N 4N1
}

The origin of large-amplitude miniature EPSCs (mEPSCs) at central synapses remains to be firmly established. Here, we show that at excitatory synapses onto magnocellular neurosecretory cells in the hypothalamus, noradrenaline induces a rapid and robust increase in mEPSC amplitude that requires $\alpha_{1}$-adrenoceptor activation but is impervious to postsynaptic manipulations that block the putative insertion of AMPA receptors. In response to noradrenaline, mEPSCs exhibit a putative multimodal amplitude histogram distribution that is not attibutable to random temporal summation, the unveiling of a quiescent synapse, or the release of large vesicles. Largeamplitude mEPSCs are sensitive to a high dose of ryanodine and are associated with an enhanced glutamate cleft concentration. Together, these data are consistent with the hypothesis that large-amplitude mEPSCs result from the synchronous release of multiple vesicles via rapid presynaptic calcium expulsion from intracellular stores.

Key words: synchronization; synaptic; glutamate; slice; norepinephrine; noradrenaline; hypothalamus

\section{Introduction}

In mammals, physiological homeostasis relies on an elegant feedback control system that is orchestrated by neural circuits in the hypothalamus. Perturbations in the "milieu interior," sensed by visceral receptors, are relayed by a plexus of noradrenergic fibers to neurons in the paraventricular nucleus of the hypothalamus (PVN) (Sawchenko and Swanson, 1981). Magnocellular neurosecretory cells (MNCs) in the PVN also receive glutamatergic input that exhibits a high frequency of quantal release, which is sensitive to noradrenaline (NA) (Boudaba et al., 2003; Gordon and Bains, 2003). Because synapses onto MNCs constitute the final integration step before the exocytosis of the hormones oxytocin and vasopressin, modulation of the efficacy of miniature EPSCs (mEPSCs) provides one mechanism through which neuroendocrine output can be enhanced (Kombian et al., 2000).

Conventional views postulate that presynaptic changes in quantal signals alter the frequency of miniature events, whereas postsynaptic changes alter the amplitude of the quantal current. Recent studies, however, challenge this precept. For instance, compelling evidence now indicates that changes in presynaptic function can increase the amplitude of the postsynaptic current (Tong and Jahr, 1994; Frerking et al., 1995; Auger et al., 1998; Liu et al., 1999; Prange and Murphy, 1999; Llano et al., 2000; Oertner et al., 2002; Sharma and Vijayaraghavan, 2003). Included in this evidence is the demonstration that the release of calcium from presynaptic stores can be extremely rapid and is capable of syn-

Received June 9, 2005; revised 0ct. 18, 2005; accepted 0ct. 18, 2005.

This work was supported by an operating grant to J.S.B. from the Canadian Institutes of Health Research (CIHR). G.R.J.G. is supported by a studentship from the Natural Sciences and Engineering Research Council. J.S.B. is a CIHR New Investigator and an Alberta Heritage Foundation for Medical Research Scholar.

Correspondence should be addressed to Jaideep S. Bains, Hotchkiss Brain Institute, University of Calgary, 3330 Hospital Drive Northwest, Calgary, Alberta, Canada, T2N 4N1. E-mail: jsbains@ucalgary.ca.

DOI:10.1523/JNEUROSCI.2378-05.2005

Copyright $\odot 2005$ Society for Neuroscience ～0270-6474/05/2511385-11\$15.00/0 chronizing the release of multiple vesicles of neurotransmitter. This results in the appearance of large-amplitude miniatures and has been shown to occur both endogenously (Llano et al., 2000) and in response to nicotinic acetylcholine receptor activation (Sharma and Vijayaraghavan, 2003). Other studies showing that larger synaptic currents are associated with an increased glutamate cleft concentration (Liu et al., 1999; Wadiche and Jahr, 2001) suggest that quantal synchronization processes may also cause the same results.

NA may be ideally suited to initiate vesicle synchronization via presynaptic calcium store activation in the PVN. Evidence suggests that functional $\alpha_{1}$-adrenoceptors are present on glutamatergic terminals synapsing onto MNCs (Boudaba et al., 2003; Gordon and Bains, 2003). Activation of these receptors elicits the release of stored calcium through a $\mathrm{G}_{\mathrm{q}}$-protein-linked intracellular cascade (Zhong and Minneman, 1999). At the neuromuscular junction, NA facilitates the synchronization of evoked release (Bukcharaeva et al., 1999) at terminals that rely on the activation of ryanodine-sensitive intracellular calcium stores for the elevation of terminal calcium to the release of acetylcholine (Narita et al., 1998; Narita et al., 2000). Furthermore, because the impact of synchronization may be constrained by the saturation of postsynaptic receptors (Tong and Jahr, 1994; Holmes, 1995; Clements, 1996; Frerking and Wilson, 1996; Auger et al., 1998), the fact that NA can also promote the insertion of postsynaptic AMPA receptors in MNCs (Gordon et al., 2005) provides an ideal complementary change to take full advantage of the enhanced presynaptic signal.

In this study, we show that NA induces a robust but transient increase in mEPSC amplitude that cannot be blocked with postsynaptic manipulations that affect AMPA receptor insertion. This increase results in a skewing of the amplitude distribution displaying putative multiple peaks, which is indicative of the synchronization of vesicles. The large-amplitude mEPSCs observed 
in NA are unlikely to occur from random temporal summation or the unveiling of a quiescent synapse or vesicle, but they are sensitive to a high dose of ryanodine. Furthermore, largeamplitude mEPSCs in NA are associated with an enhanced glutamate cleft concentration, suggesting that these events are likely multivesicular.

\section{Materials and Methods Electrophysiology}

Hypothalamic coronal slices $(300 \mu \mathrm{m})$ containing the PVN were prepared from male Sprague Dawley rats (postnatal day 21-27). The care and use of animals were in accordance with approved guidelines set forth by the University of Calgary Animal Care and Use Committee. Animals were anesthetized (sodium pentobarbital, $0.1 \mathrm{ml} / 100 \mathrm{~g}$ of body weight) and decapitated, and the brains were placed for $3 \mathrm{~min}$ in ice-cold slicing solution containing (in mM): $87 \mathrm{NaCl}, 2.5 \mathrm{KCl}, 25 \mathrm{NaHCO}_{3}, 0.5 \mathrm{CaCl}_{2}, 7$ $\mathrm{MgCl}_{2}, 1.25 \mathrm{NaH}_{2} \mathrm{PO}_{4}, 25$ glucose, 75 sucrose; saturated with $95 \%$ $\mathrm{O}_{2} / 5 \% \mathrm{CO}_{2}$. The brain was then blocked and mounted on a vibrating slicer (Leica, Nussloch, Germany) submerged in ice-cold slicing solution. Slices were incubated at $32.5^{\circ} \mathrm{C}$ in artificial CSF containing (in mM): 126 $\mathrm{NaCl}, 2.5 \mathrm{KCl}, 26 \mathrm{NaHCO}_{3}, 2 \mathrm{CaCl}_{2}, 2 \mathrm{MgCl}_{2}, 1.25 \mathrm{NaH}_{2} \mathrm{PO}_{4}, 10$ glucose; saturated with $95 \% \mathrm{O}_{2} / 5 \% \mathrm{CO}_{2}$, for a minimum of $60 \mathrm{~min}$. With an AxioskopII FS Plus upright microscope (Zeiss, Oberkochen, Germany) fitted with infrared differential interference contrast, whole-cell recordings were obtained from magnocellular PVN neurons that were confirmed by their morphology and distinct electrophysiological characteristics (Tasker and Dudek, 1991). All experimental recordings were obtained at $32.5^{\circ} \mathrm{C}$ in voltage-clamp mode and were accepted barring changes in access resistance of $>15 \%$. The intracellular solution contained (in mM): 123 potassium gluconate, $2 \mathrm{MgCl}_{2}, 8 \mathrm{NaCl}, 1$ potassium EGTA, 4 potassium ATP, and 0.3 sodium GTP buffered with $16 \mathrm{~mm}$ $\mathrm{KHCO}_{3}$. The perfusate always contained picrotoxin $(100 \mu \mathrm{M})$, to block $\mathrm{GABA}_{\mathrm{A}}$ channels, and tetrodotoxin $(1 \mu \mathrm{M})$, to block voltage-gated $\mathrm{Na}^{+}$ channels. Cells were held at $-80 \mathrm{mV}$ to block the contribution of NMDA receptors to the synaptic response.

\section{Analysis}

Gaussian fits. Amplitude histograms were plotted with 2 pA bins and appeared multimodal. Up to four modes could be fit with Gaussian functions, because larger currents were too infrequent to fit curves accurately. Gaussian curves and a sum of Gaussian curves were fit with GraphPad Prism software (version 3.0). The size of a single quantum, $q$, was calculated from the mean separation between Gaussian curves by the following expression: $q=\Sigma\left(M_{k} / k\right) / n$, where $M$ equals the mean picoampere value of each mode, $k$ equals the mode number, and $n$ equals the total number of fitted modes in the distribution.

Interevent interval fits. The time interval between events was best fit with a monoexponential equation, indicative of a stochastic Poisson release process underlying the mEPSCs in both control and NA conditions. The probability of two mEPSCs temporally summing to confer a larger amplitude mEPSC can be calculated as follows: $p_{(\text {time interval } \leq t)}=1-$ $e^{-t / \tau}$, where $t$ is the average event half-width, and $\tau$ is the $1 / e$ decay constant. We also estimated an upper limit of event summation probability with $p=t / \operatorname{avg} \Delta t$.

By using the putative multimodal amplitude distribution and assuming that every event in a given mode is actually the sum of individual events (equal to the mode number), we approximated the total number of individual events in a given amplitude distribution by deconstructing the modes (see Fig. 6D). After deconstruction, the number of observations in a mode $(X)$ was multiplied by the mode number, $k$. The number of quanta in each mode was then summed to achieve the maximum number of quantal events $\left(X_{\max }\right)$ that could occur over the time period in which the original data were collected by the following: $X_{\max }=\Sigma$ $\left[\left(X_{k}\right)(k)\right]$. This produces an avg $\Delta t$ value that is as small as possible and a summation probability that is as high as possible.

\section{Data collection and statistics}

Signals were amplified with the Multiclamp 700A amplifier (Molecular Devices, Union City, CA), low-pass filtered at $1 \mathrm{kHz}$, and digitized at 10
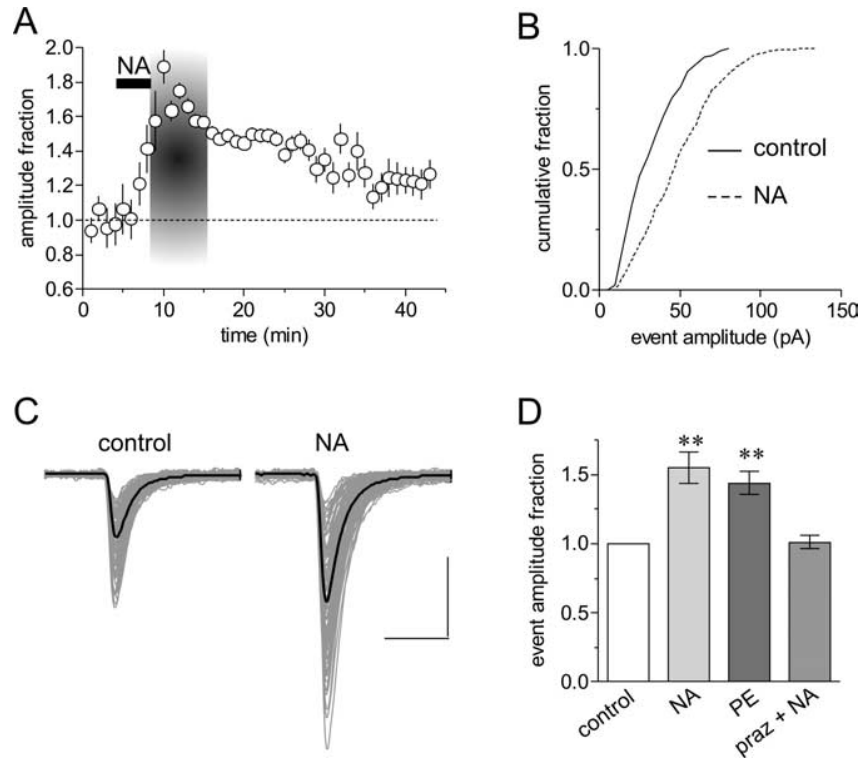

Figure 1. Noradrenaline induces a robust increase in $m E P S C$ amplitude. $\boldsymbol{A}$, Running average of mEPSC amplitude in $2 \mathrm{~min}$ bins during control and in response to a 3-5 min, $200 \mu \mathrm{m}$ bath application of NA in a single cell. The area demarcated in gray represents the time during the NA response that is compared with control values. The long-lasting increase in mEPSC amplitude caused by NA can also be observed 20 min after NA application. $\boldsymbol{B}$, Cumulative fraction plot of mEPSC amplitudes taken from control and during NA ( $p<0.01$ ); same cell as $\boldsymbol{A}$. C, Raw mEPSCS (light gray) and average mEPSC traces (solid black) from control and NA; same cell as $\boldsymbol{A}$. $\boldsymbol{D}$, Summary data depicting the change in mEPSC amplitude in response to NA $(1.55 \pm 0.11$; ${ }^{* *} p<0.01$ compared with control; $n=20$ ), the $\alpha_{1}$-adrenoceptor agonist phenylephrine (PE) $(200 \mu \mathrm{M})\left(1.44 \pm 0.08 ;{ }^{* *} p<0.01\right.$ compared with control; $\left.n=7\right)$, and NA in the presence of the $\alpha_{1}$-adrenoceptor antagonist prazosin (praz) $(10 \mu \mathrm{m})(1.03 \pm 0.06 ; p>0.05 ; n=8)$.

$\mathrm{kHz}$ with the Digidata 1322 (Molecular Devices). Data were collected (pClamp; Molecular Devices) and stored on a computer for off-line analysis with software designed to detect miniature synaptic events with a variable threshold (MiniAnalysis; Synaptosoft,Decatur, GA). Experimental values are presented as the mean \pm SEM, and statistical analyses were performed with Student's $t$ test when comparing two groups and with ANOVA with a post hoc Newman-Keuls test for comparisons across multiple groups. $p<0.05$ was accepted as statistically significant ${ }^{*} p<$ $\left.0.05 ;{ }^{* *} p<0.01\right)$.

\section{Drugs}

NA/arterenol, (R)-(-)-1-(3-hydroxyphenyl)-2-methylaminoethanol hydrochloride, prazosin, 6,7-dinitroquinoxaline-2,3(1H,4H)-dione (DNQX), GDP $\beta$ s, PMA, sucrose, 1,2-bis(2-aminophenoxy)ethane$N, N, N^{\prime}, N^{\prime}$-tetra-acetic acid tetrakis(acetoxymethyl) ester (BAPTAAM), EGTA-tetra(acetoxymethyl) ester (EGTA-AM), and ryanodine were purchased from Sigma (Oakville, Ontario, Canada). Tetrodotoxin was purchased from Alomone Labs (Jerusalem, Israel). Botulinum toxin $\mathrm{C}$ was purchased from Calbiochem (La Jolla, CA); $\gamma$-D-glutamylglycine ( $\gamma$ DGG) was purchased from Tocris Cookson (Ballwin, MO). Caffeine was a gift from Dr. Ray Turner (Hotchkiss Brain Institute, University of Calgary, Calgary, Alberta, Canada). Picrotoxin was dissolved in DMSO (final concentration, $<0.1 \%$ ). Prazosin was dissolved in methanol (final bath concentration, $<0.05 \%$ ).

\section{Results}

\section{Noradrenaline robustly increases quantal strength}

Bath application of NA $(200 \mu \mathrm{M} ; 3-5 \mathrm{~min})$ elicited a robust increase in mEPSC amplitude $(1.55 \pm 0.11 ; p<0.01 ; n=20)$ (Fig. $1 A-D)$, which was accompanied by an increase in frequency that we have characterized previously (Gordon and Bains, 2003). This is a saturating effect, and although we have observed increases in mEPSC amplitude in response to NA at $25 \mu \mathrm{M}(1.22 \pm 0.07 ; p<$ 
A
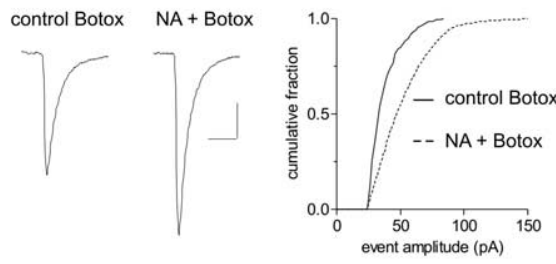

C

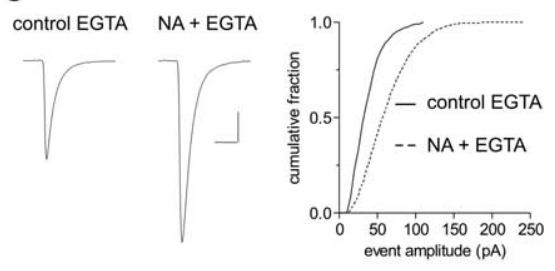

B

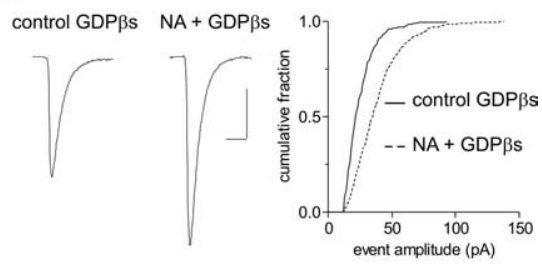

D

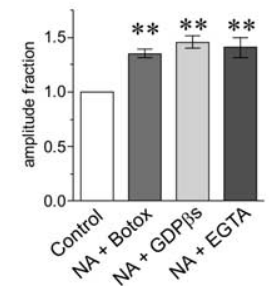

Figure 2. Postsynaptic manipulations fail to abolish the increase in $\mathrm{mEPSC}$ amplitude in response to NA. $A-C$, Average mEPSC traces on the left and cumulative fraction plots of mEPSC amplitude on the right. Inclusion of botulinum toxin $C(5 \mu \mathrm{g} / \mathrm{ml})(\boldsymbol{A})$, which blocks vesicular fusion and therefore AMPA receptor insertion, GDP $\beta \mathrm{s}(1 \mathrm{mM})(\boldsymbol{B})$, which blocks intercellular G-protein signaling, or EGTA $(10 \mathrm{~mm})(\boldsymbol{C})$, which buffers intracellular calcium, in the patch pipette failed to block the increase in mEPSC amplitude in response to NA $(p<0.01)$. D, Summary data showing that the NA effect remains in the presence of postsynaptic botulinum toxin C ( $1.35 \pm 0.04 ;{ }^{* *} p<0.01$ compared with control; $\left.n=7\right)$, GDP $\beta s\left(1.45 \pm 0.05 ;{ }^{* *} p<0.01\right.$ compared with control; $n=5)$, and elevated EGTA $(1.41 \pm 0.09 ; * * 00.01$ compared with control; $n=7)$. Botox, Botulinum toxin C.

$0.05 ; n=6)$, we have used a maximal dose to delineate the underlying mechanism. The robust increase in mEPSC amplitude is transient. It ranges from 10 to $20 \mathrm{~min}$ in length and then relaxes to a new but elevated mean $\sim 20 \%$ greater than baseline. This longlasting enhancement of mEPSC amplitude results from the release of glial-derived ATP and the subsequent insertion of postsynaptic AMPA receptors (Gordon et al., 2005). The postsynaptic effect displays a monophasic "plateau-like" increase in responsiveness to an exogenous AMPA puff and therefore is unlike the biphasic increase in mEPSC amplitude (Gordon et al., 2005). The experiments described here will focus almost exclusively on the transient increase in mEPSC amplitude caused by NA.

Consistent with previously characterized $\alpha_{1}$-adrenoceptormediated excitatory effects of NA on MNCs (Day et al., 1985; Armstrong et al., 1986; Randle et al., 1986a,b; Daftary et al., 1998; Boudaba et al., 2003; Gordon and Bains, 2003), we were able to mimic the observed increase in mEPSC amplitude with bath application of the $\alpha_{1}$-adrenoceptor agonist phenylephrine (200 $\mu \mathrm{M}$; 5 min) $(1.44 \pm 0.08 ; p<0.01 ; n=7)$ (Fig. 1D) and block the effect with the $\alpha_{1}$-adrenoceptor antagonist prazosin $(10 \mu \mathrm{M})$ $(1.03 \pm 0.06 ; p>0.05 ; n=8)($ Fig. $1 D)$. The transient increase in mEPSC amplitude is composed entirely of currents that arise from the activation of AMPA channels, because no synaptic events were observed during NA in the presence of the glutamate receptor antagonist DNQX $(n=5$; data not shown). Collectively, these data suggest that activation of $\alpha_{1}$-adrenoceptors elicits a transient and robust increase in quantal glutamate signaling.

\section{The increase in mEPSC amplitude is not blocked with postsynaptic manipulations}

Traditionally, a change in mEPSC amplitude is thought to arise from a postsynaptic modification. More recently, the insertion of postsynaptic AMPA receptors has become a widely accepted mechanism by which synapses increase their strength (Malinow and Malenka, 2002). We tested for this possibility directly by targeting soluble $\mathrm{N}$-ethylmaleimide-sensitive factor attachment protein receptor (SNARE)-dependent vesicle exocytosis in the postsynaptic cell. Inclusion of botulinum toxin $\mathrm{C}(5 \mu \mathrm{g} / \mathrm{ml})$ in our patch pipette, a compound that proteolytically cleaves the tSNARE (target membrane-associated SNARE) syntaxin (O'Connor et al., 1997), thereby blocking vesicular fusion, failed to block the transient increase in quantal amplitude caused by NA $(1.35 \pm 0.04 ; p<0.01 ; n=7)$ (Fig. $2 A, D)$. We next tested for the involvement of postsynaptic $\alpha_{1}$-adrenoceptors by inhibiting postsynaptic G-protein signaling with the inclusion of GDP $\beta$ s $(1 \mathrm{mM})$ in our internal solution (Di et al., 2003; Gordon et al., 2005). This manipulation had no impact on the transient increase in mEPSC amplitude after NA treatment $(1.45 \pm 0.05 ; p<0.01 ; n=5)$ (Fig. $2 B, D)$. We also found that the transient increase in mEPSC amplitude was not attenuated when the concentration of EGTA was increased 10-fold (from 1 to $10 \mathrm{~mm}$ ) in our internal solution $(1.41 \pm 0.09 ; p<0.01$; $n=7$ ) (Fig. 2C,D), a treatment that can block changes in postsynaptic strength in MNCs (Gordon et al., 2005). When combined, these observations argue against a role for the involvement of postsynaptic mechanisms in mediating the transient increase in mEPSC amplitude.

\section{NA enhances the skew of mEPSC amplitude distributions}

We examined the amplitude distribution of mEPSCs in control and during NA to test the hypothesis that NA acts presynaptically to synchronize the release of multiple vesicles, which would be made evident by the appearance of multiple, equidistant modes. Because numerous caveats are associated with the use of amplitude distributions in the CNS (Wahl et al., 1995; Walmsley, 1995), we used two strategies to help facilitate the detection of multiple quanta released simultaneously. First, we analyzed cells treated postsynaptically with botulinum toxin C, phosphatidylinositol-3 kinase inhibitors, or elevated EGTA to remove any confounding effects of the NA-mediated insertion of AMPA receptors on the amplitude distributions (Gordon et al., 2005). Although these treatments would be unlikely to interfere with or facilitate a presynaptic mechanism, they could provide a means for examining an isolated synchronization process. Second, we used a stringent selection procedure to focus our analysis on cells exhibiting a putative single population of events. Only cells exhibiting a unimodal event rise-time and decay-time histogram in control were used for analysis (Fig. 3A); cells exhibiting bimodal or trimodal rise-time and decay-time histograms were excluded (Fig. 3B). We reasoned that kinetically dissimilar mEPSCs might possess dissimilar mEPSC amplitudes, giving rise to multiple peaks in the amplitude histogram that do not result from the synchronous release of vesicles (Wahl et al., 1995).

Under these conditions, amplitude histograms in control were unimodal, exhibiting a characteristic rightward skew. In the presence of NA, the skew was greatly enhanced with the emergence of new equidistant modes (Fig. $4 A, B$ ): mode 1 putatively represents the response from a single quantum, and successive modes represent the response from summed quanta. Each mode was fit by a Gaussian curve, where the mean separation between 

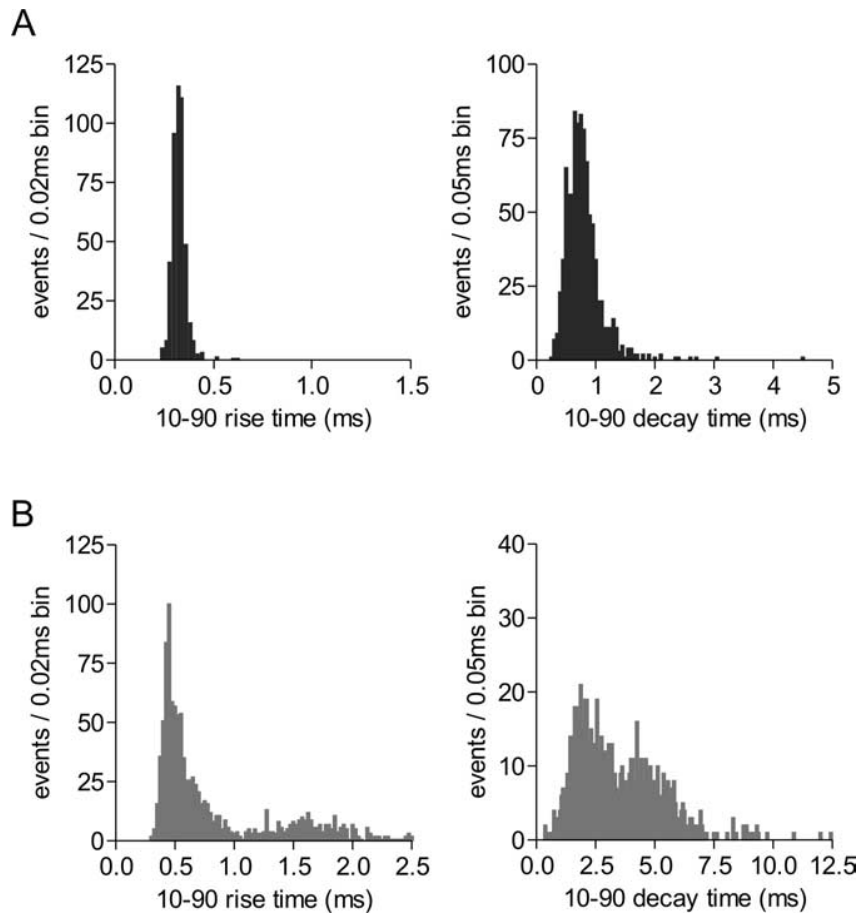

Figure 3. MNCs possess kinetically homogenous or heterogeneous mEPSCs. $\boldsymbol{A}$, Left, The 10-90 rise-time histogram; right, the 10-90 decay-time histogram from a single cell. The distributions possess a single mode, suggesting that the mEPSCs are kinetically homogenous and result from a putative single population of synapses. $\boldsymbol{B}$, Left, The $10-90$ rise-time histogram; right, the 10-90 decay-time histogram from a different cell. The distributions clearly possess more than one mode, suggesting that the mEPSCs in this cell are kinetically heterogeneous and putatively result from different populations of synapses. Cells displaying characteristics shown in $\boldsymbol{A}$ were chosen for analyses; cells exhibiting characteristics shown in $\boldsymbol{B}$ were discarded.

modes equaled the quantal size, $q(13.15 \pm 1.26 \mathrm{pA} ; n=10)$; however, the average mode SD was $32.5 \%$ of the $q$ value, which is much higher than values reported previously showing unequivocal modes (Wall and Usowicz, 1998). The SD of each mode did not increase linearly with mode number (mode $1,4.26 \pm 0.41$; mode 2, $4.30 \pm 0.78$; mode 3, $4.52 \pm 0.63$; mode 4, $6.10 \pm$ 0.48 ), a result unlike that of the neuromuscular junction (Katz, 1966) but in line with several CNS observations (Edwards et al., 1990; Larkman et al., 1991; Paulsen and Heggelund, 1994; Min and Appenteng, 1996; Wall and Usowicz, 1998). After washout of NA, the multipeaked amplitude distribution collapsed to the control form (Fig. 4C). In contrast, in cells in which postsynaptic insertion was not blocked, NA caused a rightward spread of the amplitude distribution without the clear appearance of multiple modes (Fig. $4 D, E$ ). Furthermore, when assessed $30 \mathrm{~min}$ after NA, the amplitude distribution remained slightly shifted toward larger mEPSCs compared with the control amplitude distribution (Fig. $4 F$ ), which is indicative of the long-lasting postsynaptic enhancement caused by NA (Gordon et al., 2005).

If the action of NA on presynaptic terminals can synchronize the release of vesicles, extended application of NA may not be absolutely necessary, and a more subtle technique might yield a similar result. Therefore, we hypothesized that a very brief, localized application of NA might elicit short bursts of mEPSCs and that a single burst would yield a multimodal amplitude distribution. To test this hypothesis, an electrode containing $100 \mu \mathrm{M}$ NA was placed near the dendritic region of the magnocellular neu-
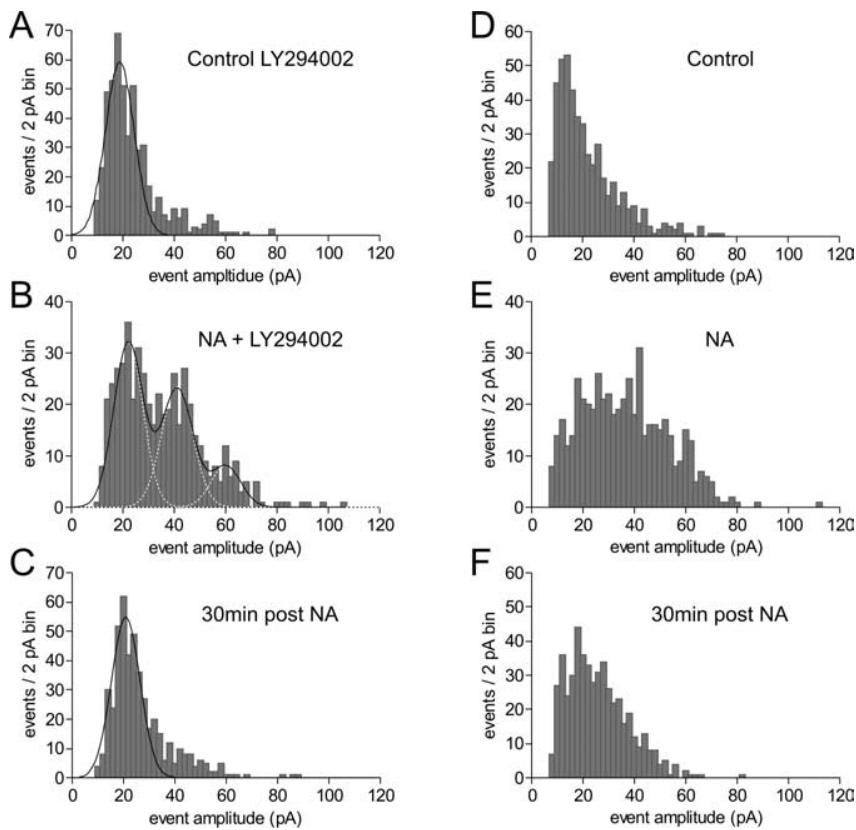

Figure 4. The mEPSC amplitude distribution in NA displays putative equidistant modes. $\boldsymbol{A}$, Control mEPSC amplitude distribution displaying a characteristic rightward skew, which cannot be fit well with a single Gaussian function. $\boldsymbol{B}$, When postsynaptic AMPA receptor insertion is blocked with the phosphatidylinositol-3 kinase inhibitor LY294002, NA enhances the skew in the amplitude distribution by the appearance of putative peaks of equal amplitude increments; same cell as $\boldsymbol{A}$. $\boldsymbol{C}$, Thirty minutes after NA (30 min post NA) treatment, the amplitude distribution returns to the control state; same cell as $\boldsymbol{A}$. $\boldsymbol{D}$, Control mEPSC amplitude distribution. $\boldsymbol{E}$, In the absence of postsynaptic blockade, NA increases the amplitude distribution skew without the clear appearance of multiple modes; same cell as $\boldsymbol{D}$. $\boldsymbol{F}$, Thirty minutes after NA treatment, the amplitude distribution remains slightly shifted to the right, indicative of the postsynaptic enhancement caused by NA; same cell as $\boldsymbol{D}$.

rons, and a picospritzer was used to apply a slow pressure pulse of NA for $\sim 2 \mathrm{~s}$. With this technique, we observed bursts of mEPSCs in 6 of 12 cells tested in this manner (supplemental Fig. 1, available at www.jneurosci.org as supplemental material). The bursts were of short duration $(5.37 \pm 1.18 \mathrm{~s} ; n=6)$ and showed a large fractional increase in mEPSC frequency $(18.09 \pm 3.9 ; p<0.01$, $n=6)$ and amplitude $(1.45 \pm 0.17 ; p<0.01 ; n=6)$. The control amplitude distributions displayed a single mode that was skewed to the right, whereas the distributions generated from mEPSCs that comprised a burst were multimodal, with an average $q$ value closely resembling that calculated for the experiments with bath application of NA $(q=15.50 \pm 1.63 \mathrm{pA} ; n=6)$ (supplemental Fig. 1, available at www.jneurosci.org as supplemental material). These data support the hypothesis that NA promotes the release of multiple vesicles and suggest that a rapid, localized action of NA can produce a result similar to that observed with bath application of the drug.

Although these data are consistent with the release of multiple vesicles for the transient increase in mEPSC amplitude, the enhanced skew in the amplitude distribution during NA may be explained equally well by the unveiling of a new synapse (Kohara et al., 2001) or vesicle (Bruns et al., 2000; Henze et al., 2002) that confers large-amplitude mEPSCs. The skew may also be explained by the random temporal summation of individual events attributable to the enhanced mEPSC frequency (Gordon and Bains, 2003), yielding true multiple peaks that do not result from a concerted synchronization process. We attempted to examine each of these possibilities as explained below. 
A

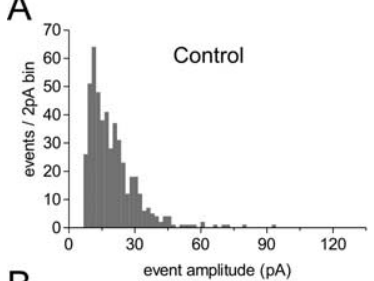

B

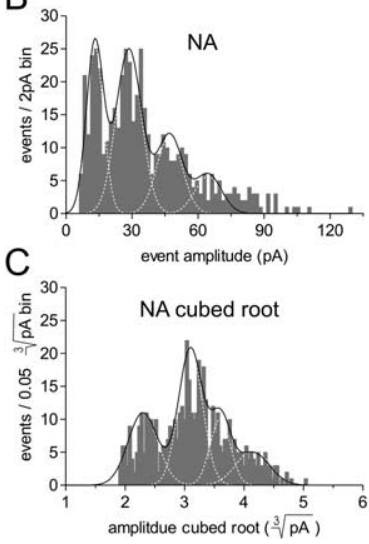

D

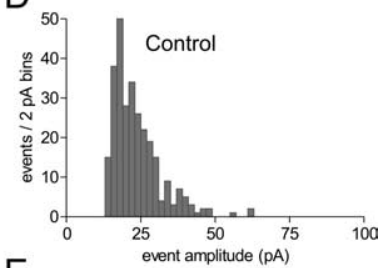

$\mathrm{E}$
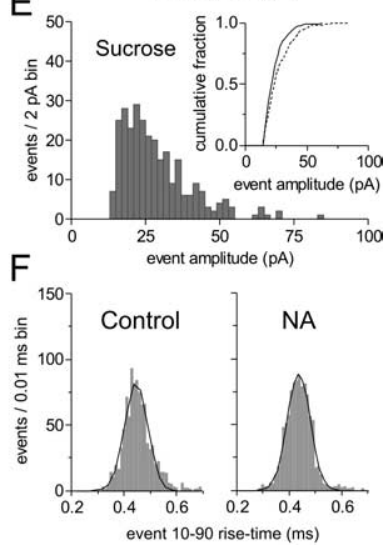

Figure 5. Large mEPSCs in NA do not result from a previously quiescent synapse or vesicle. $\boldsymbol{A}_{\text {, }}$ Control $m E P S C$ amplitude distribution. $B$, NA amplitude distribution fit with multiple Gaussians and a sum of Gaussians function; same cell as $A$. C, The cubed root transform of the NA amplitude distribution in $\boldsymbol{B}$ remains multimodal, suggesting that the release of larger vesicles by NA cannot account for the prominent skew $(n=5)$. $\boldsymbol{D}$, Control mEPSC amplitude distribution. $\boldsymbol{E}$ The secretagogue sucrose fails to increase mEPSC amplitude to the same extent as NA. This is shown by a small rightward shift in the amplitude distribution and the cumulative fraction plot of mEPSC amplitudes (inset); same cell as $\boldsymbol{D}$. $\boldsymbol{F}$, The $10-90$ rise-time histogram in control (left) remains unimodal in NA (right), suggesting that kinetically dissimilar events are not recruited during NA.

\section{The unveiling of a new synapse or vesicle does not account for} the increase in mEPSC amplitude observed in NA

To examine the possibility that NA recruits larger vesicles containing more glutamate, we used an analysis based on studies showing that the size of individual neurotransmitter vesicles at nerve terminals is not uniform (Karunanithi et al., 2002) and that the amplitude distribution of MEPSCs varies with the third power of vesicle radius (Bekkers et al., 1990). If the increase in mEPSC amplitude in response to NA is caused by the preferential release of larger vesicles containing more glutamate, the relationship between the cubed root of the individual mEPSC amplitudes in NA and the resulting transformed distribution should be Gaussian (Bruns et al., 2000). The cubed root transform, however, failed to collapse the highly skewed amplitude distribution to one that was Gaussian $(n=5)$ (Fig. $5 A-C)$.

If the transient increase in $\mathrm{mEPSC}$ amplitude observed during NA occurs by bringing a previously quiescent synapse "on-line" or, again, by triggering the release of a previously quiescent vesicle, then the action of a secretagogue should mimic the effect of NA. For this we used sucrose, which has been used extensively as a hyperosmotic stimulus to trigger release (Bekkers and Stevens, $1995)$. Bath application of sucrose (500 mM; $3 \mathrm{~min}$ ) did significantly increase the amplitude of mEPSCs $(1.15 \pm 0.02 ; p<0.01$; $n=5$ ) (Fig. $5 D, E$ ), but this effect was significantly less than that observed during NA. Moreover, we have reported previously that globally increasing release probability with phorbol esters also fails to increase mEPSC amplitude to the same extent as NA (Gordon and Bains, 2003).

Finally, if NA triggers the activation of a synapse that yields

large, kinetically dissimilar mEPSCs compared with control, this might be detected by the emergence of an additional mode in the 10-90 rise-time distribution. By using our selection process to focus the analysis on a putative single population of events, we failed to detect a distinct peak in the resulting 10-90 rise-time distribution after NA $(n=6)$ (Fig. $5 A)$. In fact, the SD of the rise-time histogram was reduced slightly in NA $(0.87 \pm 0.03 ; p<$ $0.05 ; n=6)$. Collectively, these data suggest that the transient increase in $\mathrm{MEPSC}$ amplitude caused by NA does not result from the activation of a previously quiescent synapse or vesicle.

\section{The increase in mEPSC amplitude does not occur via random} event summation

Because NA results in an enhanced mEPSC frequency (Gordon and Bains, 2003) and a multimodal amplitude distribution, we investigated the contribution of random temporal summation as an explanation for the transient increase in mEPSC amplitude. We tested for a positive correlation between fractional increases in mEPSC frequency and fractional increases in mEPSC amplitude. In 10 randomly selected cells, there was no correlation between frequency and amplitude (slope $=-0.013 \pm 0.018 ; R^{2}=$ 0.061) (Fig. 6A).

We then calculated the probability of random event overlap in two ways as follows: by $p=1-e^{t / \tau}$, where $t$ is the average event half-width, $\tau$ is the decay time constant from the monoexponential equation fit to binned $\Delta t$ mEPSC data, where $\Delta t$ is the interevent interval (Fig. $6 B$ ); and by $p=t / \operatorname{avg} \Delta t$, where $\operatorname{avg} \Delta t$ is average interevent interval over $1 \mathrm{~s}$ (Table $1, \mathrm{~Hz}$ ). These two methods represent the lower limit of event summation probability, because each assumes that no two events have summed, making the $\Delta t$ values used in each calculation as large as possible (Table 1). We then estimated an upper limit of event summation probability. By using the putative multimodal amplitude distribution and assuming that every event in a given mode is actually the sum of individual events, we can approximate the total number of individual events in a given amplitude distribution by deconstructing the modes to achieve the smallest avg $\Delta t$ value possible (Fig. $6 D$ ) (see Materials and Methods). Regardless of the method used, our analyses indicate that the probability of individual events randomly overlapping, in either control or NA, was considerably lower than the fraction of events lying outside the first mode of the amplitude distribution. This is consistent with the idea that large mEPSCs are not the result of random temporal summation.

We examined the rise-time of individual mEPSCs to determine whether large mEPSCs in NA were the result of summed quanta. First, we used high sampling $(40 \mathrm{kHz})$ and filtering $(2$ $\mathrm{kHz}$ ) rates to facilitate the detection of inflections, which would be indicative of individual quanta "stacking" on top of one another (Wall and Usowicz, 1998; Williams et al., 1998). In this analysis, however, even the largest mEPSCs displayed smooth rising phases without visible inflections (data not shown), an observation that is not consistent with summation. Second, we examined mEPSCs for a positive rise-time versus amplitude relationship. This has been interpreted as being consistent with a slight asynchrony during the release of multiple vesicles (Wall and Usowicz, 1998; Williams et al., 1998; Sharma and Vijayaraghavan, 2003). After ensuring that our normal filtering and sampling rates did not produce an artificial positive rise-time versus amplitude relationship to square current responses on a model cell (supplemental Fig. 2, available at www.jneurosci.org as supplemental material), we observed that the largest mEPSCs in NA were also the slowest; however, the slope of the rise-time versus amplitude relationship in control and NA was not signif- 
A

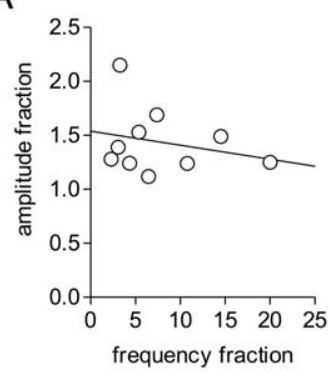

B

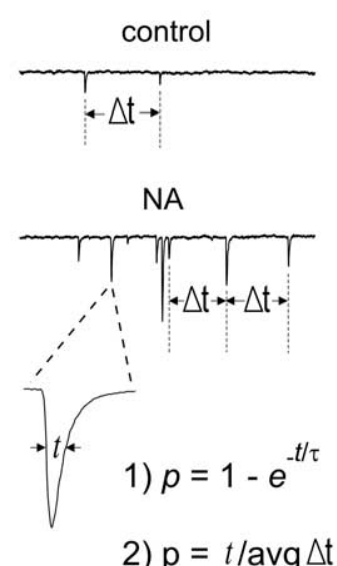

C

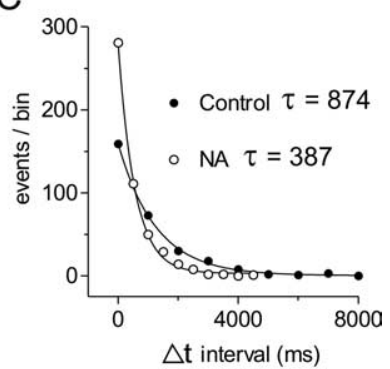

D

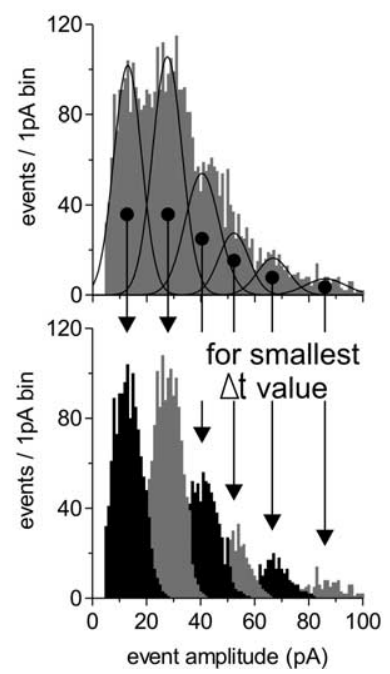

Figure 6. The increase in mEPSC amplitude by NA does not occur via random event summation. $\boldsymbol{A}$, The NA-induced fractional increase in mEPSC frequency is not related to the corresponding fractional increases in $\mathrm{mEPSC}$ amplitude (slope $=-0.013 \pm 0.018 ; R^{2}=0.061 ; n=10$ ). $\boldsymbol{B}$, Schematic depicting $\Delta t$, the interevent interval, $t$, the average event half-width, and the two formulas used to calculate the probability of random temporal summation. $\boldsymbol{C}$, Time interval between events is fit with a monoexponential equation in control ( $t=874 \mathrm{~ms}$ ) and in NA ( $t=$ $387 \mathrm{~ms}$ ), and the probability of two mEPS(s summating was calculated with $p_{\text {(time interval } \leq t)}=$ $1-e^{-t / \tau}$ in a single cell (control, $p=0.0016$; NA, $p=0.0037$ ) (Table 1). D, Amplitude histogram in NA fit with multiple Gaussian curves (top) showing the deconstruction of the raw data into the putative modes (bottom). The number of observations in a given mode after deconstruction is multiplied by the mode number to achieve the maximum number of quanta events that could have occurred in that time period, making the $\Delta t$ value as small as possible to calculate the upper limit of event summation probability (Table 1).

icantly different (control slope $=0.80 \pm 0.08 ; R^{2}=0.92$; NA slope $\left.=0.87 \pm 0.09 ; R^{2}=0.94\right)$, suggesting that the rate-limiting step governing mEPSC kinetics at a given population of synapses occurs downstream of vesicle exocytosis (Llano et al., 2000).

\section{The control amplitude distribution skews results from the release of larger vesicles}

In addition to synapse geometry (Kruk et al., 1997) and postsynaptic response variability (Faber et al., 1992), there has been strong support for a presynaptic mechanism explaining the skew in the control miniature amplitude distribution at central synapses (Frerking et al., 1995; Forti et al., 1997; Liu et al., 1999; McAllister and Stevens, 2000; Hanse and Gustafsson, 2001; Franks et al., 2003). Two popular hypotheses are that the skew (1) results from an endogenous synchronization process (Ulrich and Luscher, 1993; Paulsen and Heggelund, 1994; Llano et al., 2000) and it (2) results from variations in vesicle neurotransmitter content (Bruns et al., 2000; Karunanithi et al., 2002).

To examine the first hypothesis, we tested for endogenous

ryanodine-sensitive calcium bursts from intracellular stores, which are capable of synchronizing the release of multiple vesicles (Llano et al., 2000). For this we analyzed the amplitude of mEPSCs in response to the following: removal of extracellular calcium, which should run down the calcium supply from intracellular stores that are continually active (Llano et al., 2000); application of BAPTA-AM $(50 \mu \mathrm{M})$, a lipid permeable calcium chelator with fast binding kinetics; and application of a high-dose of ryanodine $(100 \mu \mathrm{M})$ (Llano et al., 2000; Sharma and Vijayaraghavan, 2003), which blocks these receptor-channels, thereby preventing calcium release from intracellular stores. These manipulations failed to attenuate mEPSC amplitude, however, when assessed $20 \mathrm{~min}$ after the beginning of treatment (pooled data; $0.96 \pm 0.02 ; p>0.05 ; n=14$ ) (Fig. $7 A-C)$.

To test the second hypothesis, we performed an analysis examining the amplitude distribution skew in control for a relationship to vesicle radius (Bruns et al., 2000). Taking the cubed root of the raw control amplitudes resulted in a transformed distribution that was fit well by a Gaussian curve $\left(R^{2}=0.913 \pm\right.$ $0.018 ; n=5)($ Fig. $7 D)$. These data suggest that the skew in the mEPSC amplitude distribution observed in control is not the result of a store-mediated synchronization process but instead results from larger vesicles containing more glutamate yielding larger mEPSCs.

\section{Presynaptic calcium stores are responsible for the transient increase in mEPSC amplitude}

Because there was no Gaussian relationship between the cubed root of the NA and mEPSC amplitudes (Fig. 5), we asked whether the recruitment of a store-mediated synchronization process might account for the large increase in mEPSC amplitude observed during NA. We first tested for a rise in presynaptic calcium levels. In the presence of BAPTA-AM $(50 \mu \mathrm{M})$, the increase in mEPSC amplitude caused by NA was abolished completely $(1.04 \pm 0.04 ; p>0.05 ; n=4)$ (Fig. $8 A, E$ ). We could not mimic this effect with EGTA-AM $(50 \mu \mathrm{M})$ (data not shown), although we cannot determine whether this negative outcome is the result of slower calcium binding kinetics or unsuccessful loading of EGTA-AM. To test for the presence of presynaptic calcium stores, we applied caffeine $(5 \mathrm{~mm})$ to trigger calcium release (Sharma and Vijayaraghavan, 2003). If the stores were present, this treatment would result in an elevated mEPSC frequency without necessarily mimicking rapid calcium bursts and the subsequent increase in mEPSC amplitude observed with NA (Llano et al., 2000). In accordance with this hypothesis, caffeine increased mEPSC frequency (4.05 $\pm 1.35 ; p<0.01 ; n=5$ ) (Fig. $8 B, F)$ but failed to increase mEPSC amplitude $(1.01 \pm 0.06 ; p>$ $0.05 ; n=5$ ) (Fig. $8 E$ ). We then tested for the involvement of intracellular calcium stores in the NA-induced increase in mEPSC amplitude by blocking calcium-releasing ryanodine receptor-channels with a high dose of ryanodine $(100 \mu \mathrm{M})$ (Llano et al., 2000; Sharma and Vijayaraghavan, 2003). In the presence of ryanodine, the increase in mEPSC amplitude seen during NA was attenuated $(1.18 \pm 0.04 ; p<0.01$ compared with control; $n=6)$ (Fig. $8 C, E)(p<0.01$ when compared with NA alone), suggesting that calcium release from stores is critical for the NA effect. To examine the specificity of the ryanodine block, we analyzed the change in mEPSC frequency during NA, which occurs through a separate $\alpha_{1}$-adrenoceptor-mediated mechanism and therefore should not be greatly affected by this compound (Gordon and Bains, 2003). Consistent with this idea, the increase in mEPSC frequency observed during NA in ryanodine $(6.65 \pm 2.00 ; p<$ $0.01 ; n=6)$ (Fig. $8 D, F)$ was not different $(p>0.05)$ from NA 
Table 1. Calculating the probability of random mEPSC summation in control and NA

\begin{tabular}{|c|c|c|c|c|c|c|c|c|}
\hline & \multirow{2}{*}{$\begin{array}{l}\text { mEPSC half-width } \\
t \text { (ms) }\end{array}$} & \multicolumn{2}{|c|}{$\begin{array}{l}\text { Calculated lower limit using } \\
p=1-e^{-t / \tau} \text { assuming no } \\
\text { summation present }\end{array}$} & \multicolumn{2}{|c|}{$\begin{array}{l}\text { Calculated lower limit using } \\
p=t / a v g \Delta t \text { assuming no } \\
\text { summation present }\end{array}$} & \multicolumn{2}{|c|}{$\begin{array}{l}\text { Estimated upper limit using } \\
p=t / a v g \Delta t / \text { s assuming } \\
\text { summation present }\end{array}$} & \multirow{2}{*}{$\begin{array}{l}\text { Fraction of mEPSCs }>\text { mode } \\
\text { from amplitude distribution }\end{array}$} \\
\hline & & $\tau(\mathrm{ms})$ & Probability & $\operatorname{avg} \Delta t / s(H z)$ & Probability & $\operatorname{avg} \Delta t / s(H z)$ & Probability & \\
\hline Control & $1.93 \pm 0.14$ & $582.5 \pm 180.7$ & $0.0056 \pm 0.0024$ & $2.67 \pm 0.79$ & $0.0053 \pm 0.0016$ & $3.77 \pm 0.78$ & $0.0074 \pm 0.0017$ & $0.339 \pm 0.086$ \\
\hline NA & $2.21 \pm 0.14$ & $154.8 \pm 64.14$ & $0.0226 \pm 0.0089$ & $11.21 \pm 3.61$ & $0.0259 \pm 0.0087$ & $25.67 \pm 8.86$ & $0.0595 \pm 0.0212$ & $0.646 \pm 0.047$ \\
\hline
\end{tabular}
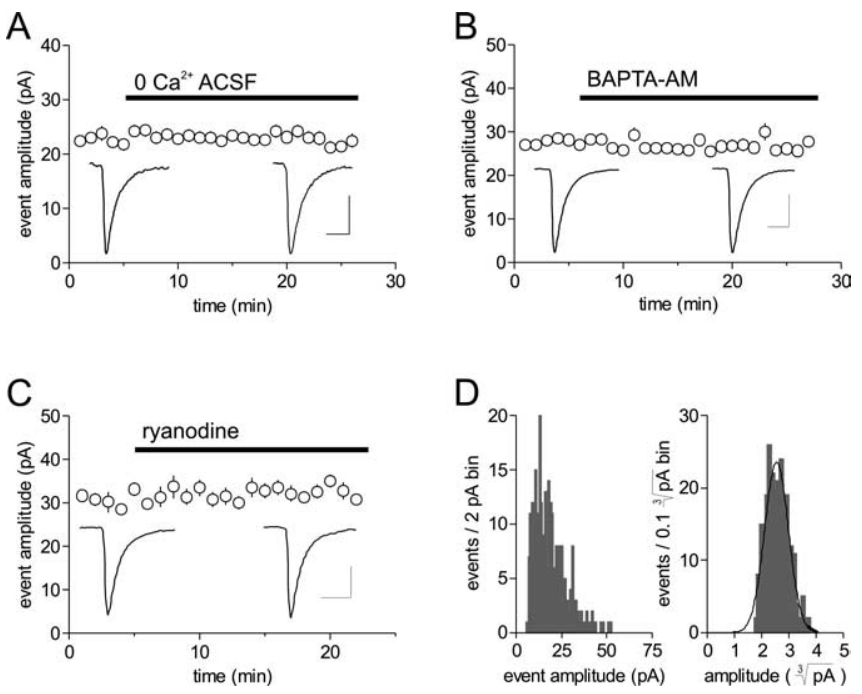

Figure 7. The control distribution skew does not result from endogenous synchronization but likely results from the release of larger vesicles containing more glutamate. Bath application of calcium-free extracellular solution $(\boldsymbol{A})$, BAPTA-AM $(50 \mu \mathrm{M})(\boldsymbol{B})$, a lipid permeable calcium chelator with fast binding kinetics, or a high dose of ryanodine $(100 \mu \mathrm{m})(\boldsymbol{C})$, which blocks ryanodine receptor-channels, failed to attenuate mEPSC amplitude when assessed 20 min after the beginning of treatment (pooled data; $0.96 \pm 0.02 ; p>0.05 ; n=14)(\boldsymbol{A}-\boldsymbol{C}$ ). D, The cubed root transform (right) of the control amplitude distribution (left) is Gaussian ( $n=5)$, suggesting that vesicles containing more glutamate underlie the control amplitude distribution skew. ACSF, Artificial CSF.

alone $(7.63 \pm 2.25 ; p<0.01 ; n=20)$. These data support the hypothesis that a rapid release of calcium from intracellular stores is necessary for the transient increase in mEPSC amplitude observed during NA.

\section{Larger mEPSCs in NA are associated with an increase in glutamate cleft concentration}

Although our experiments and the aforementioned analyses favor the fusion of multiple vesicles, they fail to show whether there is more neurotransmitter available in the synaptic cleft to bind to postsynaptic receptors. To determine whether activation of the metabotropic, presynaptic $\alpha_{1}$-adrenoceptor can increase glutamate in the cleft, we compared the effects of a high-affinity AMPA receptor antagonist, DNQX, with the effects of a low-affinity antagonist, $\gamma \mathrm{DGG}$. In contrast to DNQX, $\gamma \mathrm{DGG}$ has a transient receptor "bound time," resulting in a less effective block as glutamate concentration increases, as predicted by the laws of mass action (Liu et al., 1999). A low dose of DNQX (250 nM) uniformly blocked $\mathrm{mEPSC}$ representing the median and the 95th percentile in NA (Fig. 9A), as well as the entire amplitude distribution for both control $(p>0.05)$ and NA $(p>0.05)$ (Fig. 9C). In contrast, $\gamma$ DGG $(500 \mu \mathrm{M})$ blocked smaller mEPSCs more effectively than larger mEPSCs. This is depicted in the traces (Fig. 9B) and the normalized cumulative graphs (control, $p<0.05$; NA, $p<0.01$ ) (Fig. 9E), in which the greatest disparity is observed in the pres- ence of NA. This idea is further substantiated by the demonstration that the fractional mEPSC amplitude during $\gamma$ DGG application was larger in the presence of NA $(0.81 \pm 0.03 ; n=6)$ than in control $(0.65 \pm 0.03 ; p<0.01 ; n=5)$ or after NA $(0.62 \pm 0.02$; $p<0.01 ; n=5$ ) (Fig. 9F) (supplemental Fig. 3, available at www.jneurosci.org as supplemental material). In the latter case, the increase in mEPSC amplitude is the result of a postsynaptic enhancement of AMPA receptors (Gordon et al., 2005) and, as such, would not be expected to be less sensitive to $\gamma \mathrm{DGG}$. Importantly, these differential effects were not observed during lowdose DNQX (NA, $0.77 \pm 0.03 ; n=5$; control, $0.85 \pm 0.03 ; n=5$; $p>0.05$ ) (Fig. 9D). These data show that increases in the concentration of glutamate available to bind postsynaptic AMPA receptors contribute to the presence of large mEPSCs observed in NA.

\section{Discussion}

From the data collected in our study, we conclude that activation of $\alpha_{1}$-adrenoceptors elicits a rapid and robust increase in the amplitude of mEPSCs that is consistent with the synchronous release of multiple vesicles. This is based on several independent observations: (1) the effect was not blocked by postsynaptic treatments designed to target $\alpha_{1}$-adrenoceptors, intracellular calcium handling, or the insertion of AMPA receptors; (2) the modes in the mEPSC amplitude distributions exhibited putative equidistant modes in the presence of NA; (3) large-amplitude mEPSCs do not result from the unveiling of a previously quiescent vesicle or synapse because the amplitude distributions could not be collapsed by a cubed root transform, the effects of NA were not mimicked by a secretagogue, and the emergence of a kinetically dissimilar population of events in NA was not observed; (4) largeamplitude mEPSCs were sensitive to fast lipid permeable calcium buffering, as well as a high dose of ryanodine; and (5) the block of large mEPSCs by $\gamma$ DGG was less effective in the presence of NA.

Although both the transient and long-lasting increases in mEPSC amplitude depend on $\alpha_{1}$-adrenoceptor activation, application of the $\alpha_{1}$ antagonist prazosin in the presence of NA blocked only the transient effect, suggesting that synchronization relies on continual activation of the adrenergic receptor but insertion requires only brief activation. In contrast, the long-lasting postsynaptic change, once induced by adrenoceptor activation, is impervious to subsequent pharmacological block (Gordon et al., 2005). We currently have little evidence that these presynaptic and postsynaptic processes interact, and only that they occur simultaneously. Without postsynaptic treatment, the transient increase in mEPSC amplitude appears to be the sum of both the presynaptic and postsynaptic effects of NA. This idea is supported by the observation that the average fractional increase in mEPSC amplitude of the transient effect $(\sim 1.55)$ is the sum of a longlasting effect $(\sim 1.20)$ and the transient effect when the longlasting effect is blocked $(\sim 1.35)$.

Although the release of multiple quanta at the neuromuscular junction is thought to be responsible for the rightward skew in the amplitude distributions (Del Castillo and Katz, 1954), it has been 
difficult to determine whether this principle is valid at central synapses. Additional factors such as nonuniform postsynaptic responses attributable to intersite (Forti et al., 1997) or intrasite variance (Bekkers et al., 1990; Frerking et al., 1995) may also contribute to amplitude distributions that are skewed or exhibit multiple peaks. Because we detected kinetically disparate populations of mEPSCs in control, we could minimize intersite sources of variability with kinetic selection to examine cells exhibiting unimodal rise-time and decay-time histograms. Under these parameters, the amplitude distributions in control were still skewed, which was not the result of an endogenous synchronization process. This was shown by the inability to affect mEPSC amplitude with extracellular calcium removal, BAPTA-AM, or a high dose of ryanodine. When we examined the control amplitude distribution for a relationship to vesicle radius (Bekkers et al., 1990; Bruns et al., 2000; Karunanithi et al., 2002), we found that the control histogram, when subjected to a cubed root transformation, could be collapsed to a single Gaussian curve, consistent with larger vesicles containing more glutamate being responsible for this observation. Although obviously not an exhaustive search for an explanation of the control amplitude histogram skew, the cubed root data are also supported by the small but significant shift in the normalized cumulative fraction plot observed when control mEPSCs were treated with $\gamma$ DGG (Fig. 9E, right). This suggests that larger mEPSCs in control can also possess a greater glutamate cleft concentration.

Both precautions against (Wahl et al., 1995; Walmsley, 1995) and validations of (Larkman et al., 1997; Stratford et al., 1997) the use of amplitude histograms for detecting the release of multiple quanta have been reported. There may be several reasons why putative equidistant modes were detected in our preparation. MNCs in the PVN and supraoptic nucleus possess simple dendritic trees (Van Den Pol, 1982) and a high input resistance (1 G $\Omega$ ) (Tasker and Dudek, 1991; Bains and Ferguson, 1997), which minimize intersite variability by lessening the effects of neuronal filtering on event amplitude. We also found that blocking postsynaptic AMPA receptor insertion helped reduce the blurring of amplitude histograms. Our data show that largeamplitude mEPSCs rely on rapid calcium release from presynaptic ryanodine-sensitive receptor-channels, a process shown previously to synchronize vesicular fusion (Llano et al., 2000). Furthermore, we observed that the percentage block of mEPSC in NA by $\gamma$ DGG is less than the percentage block of mEPSCs during control, indicating that larger mEPSCs are associated with a greater concentration of cleft glutamate (Liu et al., 1999; Wadiche and Jahr, 2001). Collectively, these results support the observation of multimodal histograms and are consistent with the explanation that large mEPSCs triggered by NA are the result of multivesicular release.

Experimental manipulations that increase the probability of release directly can also increase the concentration of cleft gluta-
D
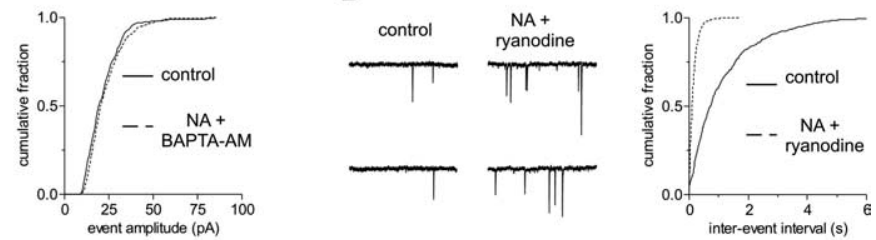

E

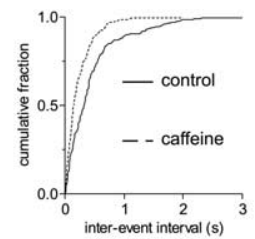

$\mathrm{F}$
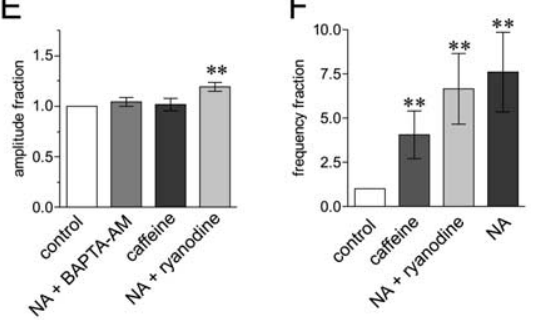

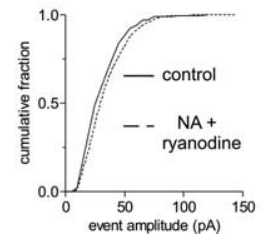

.

Figure 8. Presynaptic calcium stores mediate NA-induced vesicle synchronization. $\boldsymbol{A}$, An incubation period of $>20$ min with BAPTA-AM $(50 \mu \mathrm{m})$ completely blocked the increase in mEPSC amplitude observed in NA, shown by average mEPSC traces (right) and an amplitude cumulative fraction plot (left). $\boldsymbol{B}$, Caffeine treatment ( $5 \mathrm{~mm}$ ) increases the frequency of $\mathrm{mEPSC}$ as shown by (left) and an interevent interval cumulative fraction plot (right). $\boldsymbol{C}$, A high dose of ryanodine $1.35 ;{ }^{* *} p<0.01$ versus control; $\left.n=5\right)$, ryanodine plus NA (6.65 $\pm 2.00 ;{ }^{* *} p<0.01$ versus control; $n=6$ ), and NA alone $\left(7.63 \pm 2.25 ;{ }^{* *} p<0.01\right.$ versus control; $\left.n=20\right)$ on mEPSC frequency.

mate (Wadiche and Jahr, 2001). This classic experiment, which entails altering the $\mathrm{Ca}^{2+} / \mathrm{Mg}^{2+}$ ratio, is not well suited for our preparation because mEPSCs in MNCs are insensitive to changes in extracellular calcium concentration (Inenaga et al., 1998). This is supported further by our data showing that mEPSC amplitude remains unaltered $20 \mathrm{~min}$ after extracellular calcium removal or in the presence of BAPTA-AM. We attempted to circumvent this limitation by globally increasing release probability with hypertonic sucrose, a commonly used secretagogue (Bekkers and Stevens, 1995); however, this manipulation failed to increase the amplitude of mEPSCs to the same extent as NA, suggesting that merely increasing the probability of a single release event has no impact on the probability of synchronizing minis, and that the unveiling of a previously quiescent synapse or the preferential release of vesicles conferring large-amplitude mEPSCs are also unlikely possibilities. The increase in mEPSC amplitude observed with sucrose treatment may reflect the fusion of postsynaptic vesicles containing AMPA receptors, because the degree of the sucrose-induced enhancement is consistent with this process in MNCs (Gordon et al., 2005). The NA-triggered insertion of new AMPA receptors was also reflected in our inability to completely block the transient increase in mEPSC amplitude caused by NA in the presence of a high dose of ryanodine. This is because ryanodine should have little effect on the long-lasting enhancement of mEPSC amplitude that requires calcium influx through ATPgated P2X receptors postsynaptically (Gordon et al., 2005).

The belief that changes in the amplitude of synaptic events are determined by changes in postsynaptic efficacy alone is based on the assumption that the neurotransmitter released by one vesicle 
A
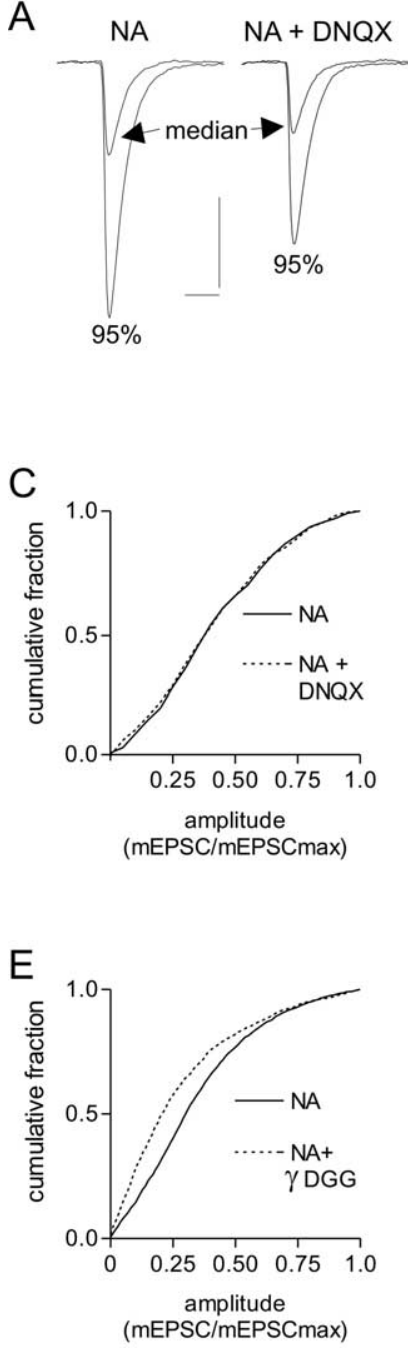

B
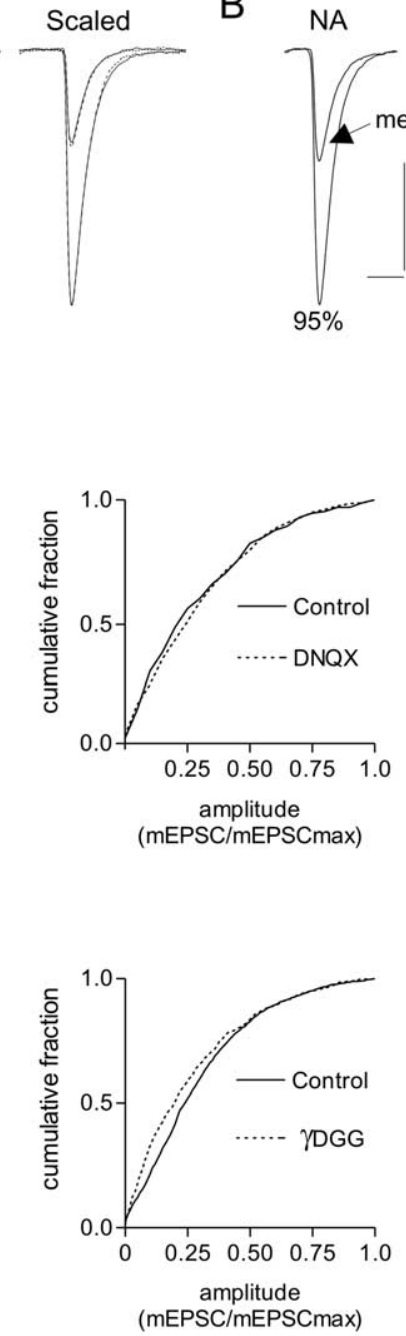

D
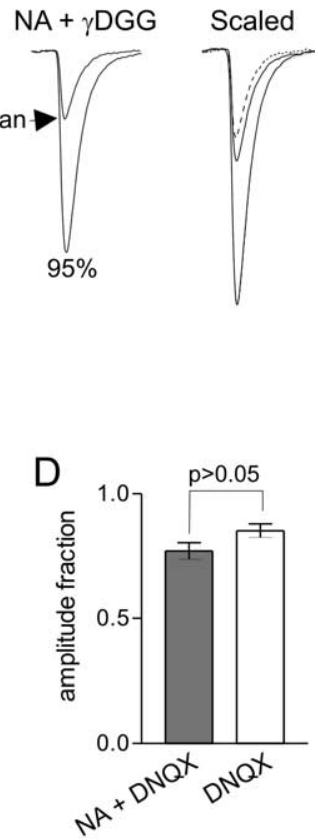

$95 \%$

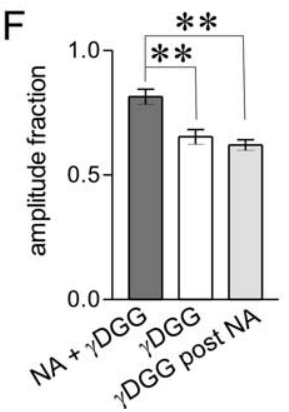

Figure 9. The increase in $\mathrm{mEPSC}$ amplitude is accompanied by a greater concentration of glutamate in the synaptic cleft. $\boldsymbol{A}$, Voltage-clamp traces from the median and 95th percentile of amplitude distributions in NA and NA plus DNQX (250 nM), a high-affinity AMPA receptor antagonist. DNQX uniformly attenuates mEPSCs from these percentiles. $\boldsymbol{B}$, Same as $\boldsymbol{A}$ for NA plus $\gamma D G G(500 \mu \mathrm{m})$, a low-affinity AMPA receptor antagonist. $\gamma D G G$ attenuates the median mEPSCs more effectively. C, Cumulative amplitude distribution normalized to the largest mEPSC for NA and NA plus DNQX (left), as well as control and control plus DNQX (right). DNQX uniformly attenuates all $\mathrm{mEPSC}$ amplitudes $(p>0.05)$. D, In DNQX, the fractional mEPSC amplitude was the same in NA $(0.77 \pm 0.03 ; n=5)$ and control $(0.85 \pm 0.03 ; p>0.05 ; n=5)$. $\boldsymbol{E}$, Cumulative amplitude distribution normalized to the largest $m E P S C$ for NA and NA plus $\gamma D G G$ (left) as well as control and control plus $\gamma D G G$ (right). $\gamma D G G$ preferentially attenuated small mEPSCs $(p<0.01)$, with the greatest disparity seen in NA. $\boldsymbol{F}$, In $\gamma \mathrm{DGG}$, the fractional mEPSC amplitude was larger during NA $(0.81 \pm 0.03 ; n=6)$ than either control $(0.65 \pm 0.03 ; p<0.01 ; n=5)$ or after NA $(0.62 \pm 0.02 ; p<0.01 ; n=5)$. Calibration: $20 \mathrm{pA}, 5 \mathrm{~ms}$.

is sufficient to saturate completely a single postsynaptic receptor field (Frerking and Wilson, 1996; Atwood and Karunanithi, 2002). Recent results, however, dispute this precept (Liu et al., 1999; Oertner et al., 2002) and suggest that the amplitude of postsynaptic currents at a given site can be regulated by altering the amount of neurotransmitter released (Tong and Jahr, 1994; Wall and Usowicz, 1998; Prange and Murphy, 1999; Wadiche and Jahr, 2001; Sharma and Vijayaraghavan, 2003). Our data cannot distinguish whether the release of multiple vesicles of glutamate onto MNCs occurs at a single release site (Tong and Jahr, 1994; Auger et al., 1998; Wadiche and Jahr, 2001; Oertner et al., 2002) or involves the synchronization of release at multiple sites (Auger and Marty, 2000; Sharma and Vijayaraghavan, 2003) with accompanying spillover and pooling of transmitter at adjacent sites. In either case, the concentration of glutamate at a single postsynaptic receptor field will be elevated. Fur-

thermore, no reported kinetic changes occur in mEPSCs when reuptake is blocked at excitatory synapses onto MNCs in the supraoptic nucleus (Oliet et al., 2001).

The cellular mechanisms responsible for vesicle synchronization may involve the production of $\mathrm{IP}_{3}$ in response to $\alpha_{1}$ adrenoceptor activation and the subsequent liberation of calcium from ryanodine-sensitive intracellular stores. Immunolocalization of $\mathrm{IP}_{3}$ receptors in the deep cerebellar nuclei and the retina (Nakanishi et al., 1991; Peng et al., 1991), as well as ryanodine receptors within basket cell terminals in the cerebellum (Llano et al., 2000), both support a role for presynaptic calcium stores at central synapses. Their functional role in terminals has been shown by observing elevations in presynaptic calcium levels in photoreceptors (Krizaj et al., 1999) and in autonomic ganglia (Peng, 1996; Smith and Cunnane, 1996) in response to caffeine and/or ryanodine treatment, whereas similar manipulations have shown an increase in the frequency (Savic and Sciancalepore, 1998) and amplitude (Sharma and Vijayaraghavan, 2003) of miniatures in hippocampal pyramidal cells. Furthermore, the release of calcium from intracellular stores can be extremely fast, resulting in the synchronization of up to 15 vesicles of neurotransmitter in a few milliseconds (Llano et al., 2000). The dose of ryanodine used here to block ryanodine receptor-channels is high but consistent with that used for previous studies examining store-mediated vesicle synchronization (Llano et al., 2000; Sharma and Vijayaraghavan, 2003). This may be necessitated by a lower affinity of ryanodine for high molecular weight isoforms in the presynaptic terminal involved in mediating the synchronization process (Llano et al., 2000).

In response to numerous physiological challenges including dehydration, hemorrhage, stress, lactation, and parturition, NA is released in the PVN (Pacak et al., 1995) to increase neuroendocrine and autonomic output (Day et al., 1984; Pacak et al., 1995; Cole and Sawchenko, 2002). Highfrequency stimulation of glutamatergic fibers onto MNCs in the supraoptic nucleus has also been shown to increase the amplitude of mEPSCs and increase postsynaptic spiking (Kombian et al., 2000). Our data offer a physiological context for the rapid amplification of hormone output from the posterior pituitary during periods of high demand. Such a mechanism would work in conjunction with changes in the gating of postsynaptic osmosensitive channels (Oliet and Bourque, 1993) or membrane depolarizations resulting from the direct actions of neuromodulators such as angiotensin II on postsynaptic ion channels (Li and Ferguson, 1996), such that large-amplitude quantal events would be more successful at generating postsynaptic firing when MNCs idle closer to sodium spike threshold. 


\section{References}

Armstrong WE, Gallagher MJ, Sladek CD (1986) Noradrenergic stimulation of supraoptic neuronal activity and vasopressin release in vitro: mediation by an alpha 1-receptor. Brain Res 365:192-197.

Atwood HL, Karunanithi S (2002) Diversification of synaptic strength: presynaptic elements. Nat Rev Neurosci 3:497-516.

Auger C, Marty A (2000) Quantal currents at single-site central synapses. J Physiol (Lond) 526:3-11.

Auger C, Kondo S, Marty A (1998) Multivesicular release at single functional synaptic sites in cerebellar stellate and basket cells. J Neurosci 18:4532-4547.

Bains JS, Ferguson AV (1997) Nitric oxide regulates NMDA-driven GABAergic inputs to type I neurones of the rat paraventricular nucleus. J Physiol (Lond) 499:733-746.

Bekkers JM, Stevens CF (1995) Quantal analysis of EPSCs recorded from small numbers of synapses in hippocampal cultures. J Neurophysiol 73:1145-1156.

Bekkers JM, Richerson GB, Stevens CF (1990) Origin of variability in quantal size in cultured hippocampal neurons and hippocampal slices. Proc Natl Acad Sci USA 87:5359-5362.

Boudaba C, Di S, Tasker JG (2003) Presynaptic noradrenergic regulation of glutamate inputs to hypothalamic magnocellular neurones. J Neuroendocrinol 15:803-810.

Bruns D, Riedel D, Klingauf J, Jahn R (2000) Quantal release of serotonin. Neuron 28:205-220.

Bukcharaeva EA, Kim KC, Moravec J, Nikolsky EE, Vyskocil F (1999) Noradrenaline synchronizes evoked quantal release at frog neuromuscular junctions. J Physiol (Lond) 517:879-888.

Clements JD (1996) Transmitter time course in the synaptic cleft: its role in central synaptic function. Trends Neurosci 19:163-171.

Cole RL, Sawchenko PE (2002) Neurotransmitter regulation of cellular activation and neuropeptide gene expression in the paraventricular nucleus of the hypothalamus. J Neurosci 22:959-969.

Daftary SS, Boudaba C, Szabo K, Tasker JG (1998) Noradrenergic excitation of magnocellular neurons in the rat hypothalamic paraventricular nucleus via intranuclear glutamatergic circuits. J Neurosci 18:10619-10628.

Day TA, Ferguson AV, Renaud LP (1984) Facilitatory influence of noradrenergic afferents on the excitability of rat paraventricular nucleus neurosecretory cells. J Physiol (Lond) 355:237-249.

Day TA, Randle JC, Renaud LP (1985) Opposing alpha- and betaadrenergic mechanisms mediate dose-dependent actions of noradrenaline on supraoptic vasopressin neurones in vivo. Brain Res 358:171-179.

Del Castillo J, Katz B (1954) Quantal components of the end-plate potential. J Physiol (Lond) 124:560-573.

Di S, Malcher-Lopes R, Halmos KC, Tasker JG (2003) Nongenomic glucocorticoid inhibition via endocannabinoid release in the hypothalamus: a fast feedback mechanism. J Neurosci 23:4850-4857.

Edwards FA, Konnerth A, Sakmann B (1990) Quantal analysis of inhibitory synaptic transmission in the dentate gyrus of rat hippocampal slices: a patch-clamp study. J Physiol (Lond) 430:213-249.

Faber DS, Young WS, Legendre P, Korn H (1992) Intrinsic quantal variability due to stochastic properties of receptor-transmitter interactions. Science 258:1494-1498.

Forti L, Bossi M, Bergamaschi A, Villa A, Malgaroli A (1997) Loose-patch recordings of single quanta at individual hippocampal synapses. Nature 388:874-878.

Franks KM, Stevens CF, Sejnowski TJ (2003) Independent sources of quantal variability at single glutamatergic synapses. J Neurosci 23:3186-3195.

Frerking M, Wilson M (1996) Saturation of postsynaptic receptors at central synapses? Curr Opin Neurobiol 6:395-403.

Frerking M, Borges S, Wilson M (1995) Variation in GABA mini amplitude is the consequence of variation in transmitter concentration. Neuron 15:885-895.

Gordon GRJ, Bains JS (2003) Priming of excitatory synapses by $\alpha 1$ adrenoceptor-mediated inhibition of group III metabotropic glutamate receptors. J Neurosci 23:6223-6231.

Gordon GRJ, Baimoukhametova DV, Hewitt SA, Rajapaksha WRAKJS, Fisher TE, Bains JS (2005) Noradrenaline triggers release of glial ATP to increase postsynaptic efficacy. Nat Neurosci 8:1078-1086.

Hanse E, Gustafsson B (2001) Quantal variability at glutamatergic synapses in area CA1 of the rat neonatal hippocampus. J Physiol (Lond) 531:467-480.
Henze DA, McMahon DB, Harris KM, Barrionuevo G (2002) Giant miniature EPSCs at the hippocampal mossy fiber to CA3 pyramidal cell synapse are monoquantal. J Neurophysiol 87:15-29.

Holmes WR (1995) Modeling the effect of glutamate diffusion and uptake on NMDA and non-NMDA receptor saturation. Biophys J 69:1734-1747.

Inenaga K, Honda E, Hirakawa T, Nakamura S, Yamashita H (1998) Glutamatergic synaptic inputs to mouse supraoptic neurons in calcium-free medium in vitro. J Neuroendocrinol 10:1-7.

Karunanithi S, Marin L, Wong K, Atwood HL (2002) Quantal size and variation determined by vesicle size in normal and mutant Drosophila glutamatergic synapses. J Neurosci 22:10267-10276.

Katz B (1966) Nerve, muscle and synapse. New York: McGraw-Hill.

Kohara K, Ogura A, Akagawa K, Yamaguchi K (2001) Increase in number of functional release sites by cyclic AMP-dependent protein kinase in cultured neurons isolated from hippocampal dentate gyrus. Neurosci Res 41:79-88.

Kombian SB, Hirasawa M, Mouginot D, Chen X, Pittman QJ (2000) Shortterm potentiation of miniature excitatory synaptic currents causes excitation of supraoptic neurons. J Neurophysiol 83:2542-2553.

Krizaj D, Bao JX, Schmitz Y, Witkovsky P, Copenhagen DR (1999) Caffeine-sensitive calcium stores regulate synaptic transmission from retinal rod photoreceptors. J Neurosci 19:7249-7261.

Kruk PJ, Korn H, Faber DS (1997) The effects of geometrical parameters on synaptic transmission: a Monte Carlo simulation study. Biophys J 73:2874-2890.

Larkman A, Stratford K, Jack J (1991) Quantal analysis of excitatory synaptic action and depression in hippocampal slices. Nature 350:344-347.

Larkman AU, Jack JJ, Stratford KJ (1997) Assessment of the reliability or amplitude histograms from excitatory synapses in rat hippocampal CA1 in vitro. J Physiol (Lond) 505:443-456.

LiZ, Ferguson AV (1996) Electrophysiological properties of paraventricular magnocellular neurons in rat brain slices: modulation of IA by angiotensin II. Neuroscience 71:133-145.

Liu G, Choi S, Tsien RW (1999) Variability of neurotransmitter concentration and nonsaturation of postsynaptic AMPA receptors at synapses in hippocampal cultures and slices. Neuron 22:395-409.

Llano I, Gonzalez J, Caputo C, Lai FA, Blayney LM, Tan YP, Marty A (2000) Presynaptic calcium stores underlie large-amplitude miniature IPSCs and spontaneous calcium transients. Nat Neurosci 3:1256-1265.

Malinow R, Malenka RC (2002) AMPA receptor trafficking and synaptic plasticity. Annu Rev Neurosci 25:103-126.

McAllister AK, Stevens CF (2000) Nonsaturation of AMPA and NMDA receptors at hippocampal synapses. Proc Natl Acad Sci USA 97:6173-6178.

Min MY, Appenteng K (1996) Multimodal distribution of amplitudes of miniature and spontaneous EPSPs recorded in rat trigeminal motoneurones. J Physiol (Lond) 494:171-182.

Nakanishi S, Maeda N, Mikoshiba K (1991) Immunohistochemical localization of an inositol 1,4,5-trisphosphate receptor, P400, in neural tissue: studies in developing and adult mouse brain. J Neurosci 11:2075-2086.

Narita K, Akita T, Osanai M, Shirasaki T, Kijima H, Kuba K (1998) $\mathrm{A} \mathrm{Ca}^{2+}$. induced $\mathrm{Ca}^{2+}$ release mechanism involved in asynchronous exocytosis at frog motor nerve terminals. J Gen Physiol 112:593-609.

Narita K, Akita T, Hachisuka J, Huang S, Ochi K, Kuba K (2000) Functional coupling of $\mathrm{Ca}(2+)$ channels to ryanodine receptors at presynaptic terminals: amplification of exocytosis and plasticity. J Gen Physiol 115:519-532.

O’Connor V, Heuss C, De Bello WM, Dresbach T, Charlton MP, Hunt JH, Pellegrini LL, Hodel A, Burger MM, Betz H, Augustine GJ, Schafer T (1997) Disruption of syntaxin-mediated protein interactions blocks neurotransmitter secretion. Proc Natl Acad Sci USA 94:12186-12191.

Oertner TG, Sabatini BL, Nimchinsky EA, Svoboda K (2002) Facilitation at single synapses probed with optical quantal analysis. Nat Neurosci 5:657-664.

Oliet SH, Bourque CW (1993) Mechanosensitive channels transduce osmosensitivity in supraoptic neurons. Nature 364:341-343.

Oliet SH, Piet R, Poulain DA (2001) Control of glutamate clearance and synaptic efficacy by glial coverage of neurons. Science 292:923-926.

Pacak K, Palkovits M, Kvetnansky R, Yadid G, Kopin IJ, Goldstein DS (1995) Effects of various stressors on in vivo norepinephrine release in the hypothalamic paraventricular nucleus and on the pituitary-adrenocortical axis. Ann NY Acad Sci 771:115-130. 
Paulsen O, Heggelund P (1994) The quantal size at retinogeniculate synapses determined from spontaneous and evoked EPSCs in guinea-pig thalamic slices. J Physiol (Lond) 480:505-511.

Peng Y (1996) Ryanodine-sensitive component of calcium transients evoked by nerve firing at presynaptic nerve terminals. J Neurosci 16:6703-6712.

Peng YW, Sharp AH, Snyder SH, Yau KW (1991) Localization of the inositol 1,4,5-trisphosphate receptor in synaptic terminals in the vertebrate retina. Neuron 6:525-531.

Prange O, Murphy TH (1999) Analysis of multiquantal transmitter release from single cultured cortical neuron terminals. J Neurophysiol 81:1810-1817.

Randle JC, Bourque CW, Renaud LP (1986a) Alpha 1-adrenergic receptor activation depolarizes rat supraoptic neurosecretory neurons in vitro. Am J Physiol 251:R569-R574.

Randle JC, Day TA, Jhamandas JH, Bourque CW, Renaud LP (1986b) Neuropharmacology of supraoptic nucleus neurons: norepinephrine and gamma-aminobutyric acid receptors. Fed Proc 45:2312-2317.

Savic N, Sciancalepore M (1998) Intracellular calcium stores modulate miniature GABA-mediated synaptic currents in neonatal rat hippocampal neurons. Eur J Neurosci 10:3379-3386.

Sawchenko PE, Swanson LW (1981) Central noradrenergic pathways for the integration of hypothalamic neuroendocrine and autonomic responses. Science 214:685-687.

Sharma G, Vijayaraghavan S (2003) Modulation of presynaptic store calcium induces release of glutamate and postsynaptic firing. Neuron 38:929-939.

Smith AB, Cunnane TC (1996) Ryanodine-sensitive calcium stores involved in neurotransmitter release from sympathetic nerve terminals of the guinea-pig. J Physiol (Lond) 497:657-664.
Stratford KJ, Jack JJ, Larkman AU (1997) Calibration of an autocorrelationbased method for determining amplitude histogram reliability and quantal size. J Physiol (Lond) 505:425-442.

Tasker JG, Dudek FE (1991) Electrophysiological properties of neurones in the region of the paraventricular nucleus in slices of rat hypothalamus. J Physiol (Lond) 434:271-293.

Tong G, Jahr CE (1994) Multivesicular release from excitatory synapses of cultured hippocampal neurons. Neuron 12:51-59.

Ulrich D, Luscher HR (1993) Miniature excitatory synaptic currents corrected for dendritic cable properties reveal quantal size and variance. J Neurophysiol 69:1769-1773.

Van Den Pol AN (1982) The magnocellular and parvocellular paraventricular nucleus of rat: intrinsic organization. J Comp Neurol 206:317-345.

Wadiche JI, Jahr CE (2001) Multivesicular release at climbing fiberPurkinje cell synapses. Neuron 32:301-313.

Wahl LM, Stratford KJ, Larkman AU, Jack JJ (1995) The variance of successive peaks in synaptic amplitude histograms: effects of inter-site differences in quantal size. Proc Biol Sci 262:77-85.

Wall MJ, Usowicz MM (1998) Development of the quantal properties of evoked and spontaneous synaptic currents at a brain synapse. Nat Neurosci 1:675-682.

Walmsley B (1995) Interpretation of "quantal" peaks in distributions of evoked synaptic transmission at central synapses. Proc Biol Sci 261:245-250.

Williams SR, Buhl EH, Mody I (1998) The dynamics of synchronized neurotransmitter release determined from compound spontaneous IPSCs in rat dentate granule neurones in vitro. J Physiol (Lond) 510:477-497.

Zhong H, Minneman KP (1999) Alphal-adrenoceptor subtypes. Eur J Pharmacol 375:261-276. 